\title{
Cycling State that Can Lead to Glassy Dynamics in Intracellular Transport
}

\author{
Monika Scholz, ${ }^{1,2}$ Stanislav Burov, ${ }^{3}$ Kimberly L. Weirich, ${ }^{1,2}$ Björn J. Scholz, ${ }^{4,5}$ S. M. Ali Tabei, ${ }^{7}$ \\ Margaret L. Gardel,${ }^{1,2,6}$ and Aaron R. Dinner ${ }^{1,2,8, *}$ \\ ${ }^{1}$ James Franck Institute, the University of Chicago, Chicago, Illinois 60637, USA \\ ${ }^{2}$ Institute for Biophysical Dynamics, the University of Chicago, Chicago, Illinois 60637, USA \\ ${ }^{3}$ Department of Physics, Bar-Ilan University, Ramat-Gan 5290002, Israel \\ ${ }^{4}$ Enrico Fermi Institute, the University of Chicago, Chicago, Illinois 60637, USA \\ ${ }^{5}$ Kavli Institute for Cosmological Physics, the University of Chicago, Chicago, Illinois 60637, USA \\ ${ }^{6}$ Department of Physics, the University of Chicago, Chicago, Illinois 60637, USA \\ ${ }^{7}$ Physics Department, University of Northern Iowa, Cedar Falls, Iowa 50614, USA \\ ${ }^{8}$ Department of Chemistry, the University of Chicago, Chicago, Illinois 60637, USA \\ (Received 31 March 2015; revised manuscript received 11 February 2016; published 31 March 2016)
}

\begin{abstract}
Power-law dwell times have been observed for molecular motors in living cells, but the origins of these trapped states are not known. We introduce a minimal model of motors moving on a two-dimensional network of filaments, and simulations of its dynamics exhibit statistics comparable to those observed experimentally. Analysis of the model trajectories, as well as experimental particle tracking data, reveals a state in which motors cycle unproductively at junctions of three or more filaments. We formulate a master equation for these junction dynamics and show that the time required to escape from this vortexlike state can account for the power-law dwell times. We identify trends in the dynamics with the motor valency for further experimental validation. We demonstrate that these trends exist in individual trajectories of myosin II on an actin network. We discuss how cells could regulate intracellular transport and, in turn, biological function by controlling their cytoskeletal network structures locally.
\end{abstract}

DOI: 10.1103/PhysRevX.6.011037

\section{INTRODUCTION}

Individual microscopic particles (beads [1,2] or fluorescently labeled molecules [3-5]) can now be tracked in cells. These studies reveal complex dynamics [6-8]. The resulting trajectories can be treated as random walks, and quantitative analysis of their statistics can provide insights into underlying mechanisms $[9,10]$. Often, the mean-square displacement (MSD) exhibits a power-law (typically sublinear) dependence on the separation in time between two observations, known as the lag time $(\Delta)$ [9-15]. In certain cases $[9,14]$, the MSD also decays as the amount of data included in averages (the measurement time $T$ ) increases; this trend indicates a power-law distribution of dwell times and is known as "aging" in theories of glassy dynamics [16].

These power-law statistics can have important biological implications $[9,17]$. For example, a recent study shows that the anomalous dynamics observed for insulin secretory vesicles (granules) can account for the biphasic kinetics of

\footnotetext{
*To whom all correspondence should be addressed. dinner@uchicago.edu

Published by the American Physical Society under the terms of the Creative Commons Attribution 3.0 License. Further distribution of this work must maintain attribution to the author(s) and the published article's title, journal citation, and DOI.
}

Subject Areas: Biological Physics, Interdisciplinary Physics, Statistical Physics insulin release [9] without the need to invoke separate pools of granules, as previously [18]. In particular, the sustained release relies on the glassy dynamics. Glassy dynamics are often interpreted in terms of trapping in local minima of an energy landscape with an exponential or power-law distribution of depths $[19,20]$. However, how such a landscape could arise from typical biomolecular interactions is unclear. Crowding is insufficient, as it results in standard Brownian motion but with a reduced diffusion coefficient [21]. Because the moving vesicles are associated with molecular motors, which consume cellular energy stores (nucleotide triphosphates) for directed motion along cytoskeletal filaments, other, intrinsically nonequilibrium mechanisms of generating these statistics may exist.

In the case of insulin release, the vesicles have both kinesin and dynein associated with them [22], which walk in opposite directions on microtubules [23]. More generally, many cytoskeletal assemblies in cells have multiple motors associated with them (Fig. 1). Thus, it is natural to ask if glassy dynamics could arise from a tug of war. Mathematical models of competing motors have been formulated and show that, depending on the number of motors, their properties, and their binding affinities, different regimes of transport kinetics can be accessed [24,25]. While the tugof-war model can explain bidirectional transport in a diverse set of biological systems [26-29], standard formulations 


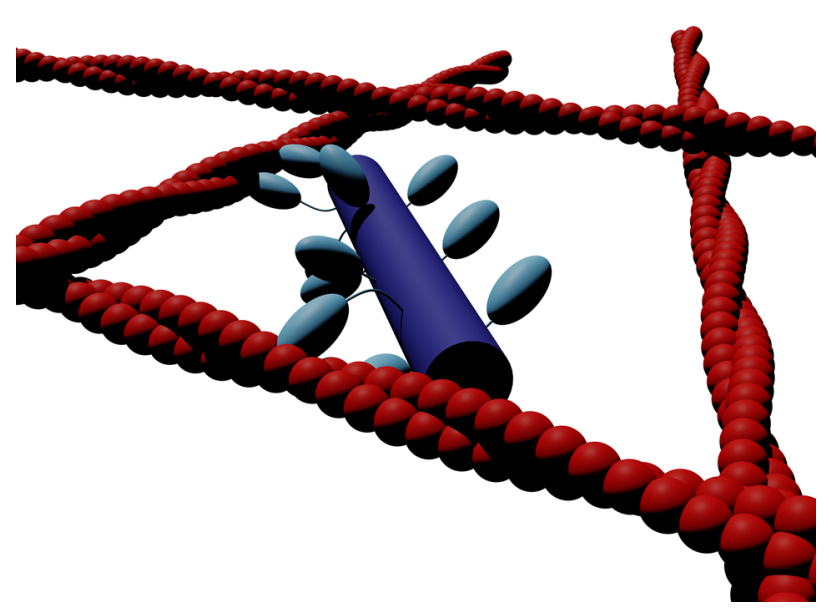

FIG. 1. Schematic of a cytoskeletal assembly composed of multiple molecular motors. Drawing is to scale for actin filaments (red) and myosin motors (blue).

cannot account for aging because exponential dwell times are assumed. Moreover, the tug of war is essentially a single-filament mechanism. Cytoskeletal networks contain geometric structures that involve multiple filaments (e.g., junctions), and these could support other dynamics.

In this paper, we investigate the dynamics of a minimal model of a motor that can make multiple attachments to a two-dimensional network of filaments. Using simulations, we show that such a model can exhibit glassy dynamics, and we discover that the long-time correlations in this model result from vortexlike trajectories that motors follow when three or more filaments cross to form a circuit. This represents a new mechanism for trapping that does not require individual motor heads stalling, and we term it the "cycling state." We obtain average flows for idealized junction geometries from a master equation analysis and show that trapping in vortexlike cycling states can give rise to glassy, nonergodic dynamics like those observed in experiments. We analyze experimental particle tracking data to demonstrate the presence of the cycling state in measured trajectories and show that it relates to exponents that quantify aging. Broader implications for biological function are discussed.

\section{MOLECULAR SIMULATIONS}

We model filaments as randomly oriented line segments in a plane. The length of each filament is drawn from a normal distribution with a maximum length $\ell$ and standard deviation $\sigma_{\ell}$. We associate the polarity of filament $i$ with a fixed unit vector $\vec{e}_{i}$. The filaments are static, and thus represent experimental situations in which cytoskeletal rearrangements are slow in comparison with the period over which motor transport is measured. For simplicity, we also neglect heterogeneities in the composition of the filaments and the solution environment, which can lead to complex dynamics [25].
We are interested in cases in which many molecular motors act in concert-e.g., a vesicle with several protein motors attached or a myosin minifilament. We refer to our model of such an assembly as "a motor." A motor is a point particle that can bind up to $M$ filaments at once and move along them as follows. We separate the binding process into two steps (Figs. 2(a) and 2(b); see Supplemental Material and Fig. S1 therein for an alternative scheme [30]). First, we distribute the $M$ possible attachments for a motor among filaments with probability proportional to $b_{i}=\exp \left[-\left(3 d_{i} / 2 s\right)^{2}\right]$, where $d_{i}$ is the shortest distance between the motor and filament $i$, and $s$ is a parameter that sets the interaction length scale. This probability is normalized by the sum $\sum_{i} b_{i}$ over all filaments within a distance $3 s$ of the motor. Then, we determine if each such interaction exerts force to move the motor (henceforth, "active") with probability $b_{i}$ or not ("inactive") with probability $1-b_{i}$. We denote the number of active attachments to filament $i$ by $k_{i}$. To determine the change in position of the motor, we compute the vector $\vec{v}=\sum_{i} k_{i} \vec{e}_{i}$ [orange in Fig. 2(b)]. This choice is consistent with measurements that show that motor velocities increase with head numbers [31]. We project $\vec{v}$ onto all the filaments with at least one active attachment and add to the motor position the projection with the maximum magnitude scaled by the time step [Figs. 2(c) and 2(d)]. This projection rule ensures that the motor moves along rather than off (a) Identify filaments in
binding radius

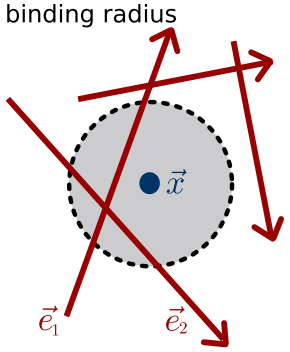

(c) Velocity projection

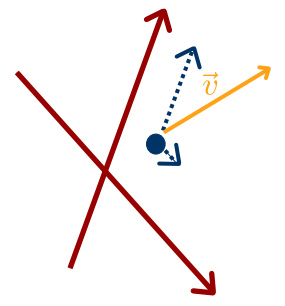

(b) Binding to filaments with distance-dependent

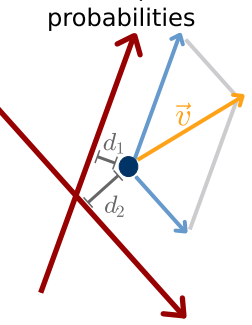

(d) Movement along maximal projection

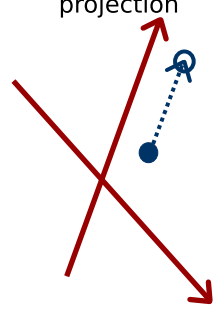

FIG. 2. Schematic of the molecular simulation procedure. (a) The motor (blue dot) can associate with filaments (red vectors) within a defined binding radius. (b) Active attachments are assigned stochastically in a distance-dependent fashion. The velocity vector that results from each active attachment moving a step along its filament is computed. (c) The velocity vector is projected onto the filaments. (d) The motor takes a step with size proportional to the magnitude of the largest velocity projection, along the associated filament. 
TABLE I. Simulation parameters.

\begin{tabular}{lc}
\hline \hline Parameter & Value \\
\hline Filament density per unit area & 1 \\
Filament length, $\ell$ & 5 \\
Filament standard deviation, $\sigma_{\ell}$ & 5 \\
Binding radius, $s$ & 0.01 \\
Total number of binding sites, $M$ & $50-100$ \\
Time step, $d t$ & 0.001 \\
Total number of steps, $T$ & $10^{5}$ \\
Trials & 2000 \\
\hline \hline
\end{tabular}

filaments. It also gives rise to an effective force-velocity dependence, with opposing parallel velocities canceling each other. We assume that forces that are directed orthogonally do not create a load on the motor. For the simple scenario of orthogonal filaments, this scheme simplifies to a step in the direction of the filament with the highest number of active binding interactions (i.e., a majority rule). The projection rule implies that binding sites do not detach under load; rather, they stay bound and contribute to the overall velocity vector. Simulations relaxing the projection rule to a simple net velocity calculation show only minor differences (Fig. S2 in Supplemental Material [30]). Simulations with stochastic selection between projections with probabilities proportional to their magnitudes also yielded similar results.

The values of the simulation parameters are given in Table I. While the model is general, we choose the values to be roughly consistent with actin and myosin to ensure that we study a physically reasonable regime. To this end, we assume a myosin speed of $1 \mu \mathrm{m} / \mathrm{s}$, which is in the range of speeds reported from in vitro and in vivo studies of various classes of myosin [32-34]. We associate our unit length with $1 \mu \mathrm{m}$, such that the average filament length is $5 \mu \mathrm{m}$. The binding radius of the motor is then about $10 \mathrm{~nm}$, and a single time step of the simulation is $0.1 \mathrm{~s}$. Although the properties of actual molecular motors vary substantially, our conclusions are robust to parameter choices that range over an order of magnitude (Fig. 3).

We simulate the model according to the rules above and calculate the time-averaged MSD for the resulting trajectories:

$$
\left\langle\overline{\vec{R}(T, \Delta)^{2}}\right\rangle=\frac{1}{T-\Delta} \int_{0}^{T-\Delta}[\vec{x}(t+\Delta)-\vec{x}(t)]^{2} d t,
$$

where $\vec{x}(t)$ is the position of a motor at time $t$. We plot the time-averaged MSD as a function of lag time $(\Delta)$ in Fig. 3(a). We use the time-averaged MSD to make connection with biological experiments that typically have a limited number of trajectories [35]. It is important to note that the time- and ensemble-averaged MSDs can exhibit different scalings when the process of interest is nonergodic [36-40].
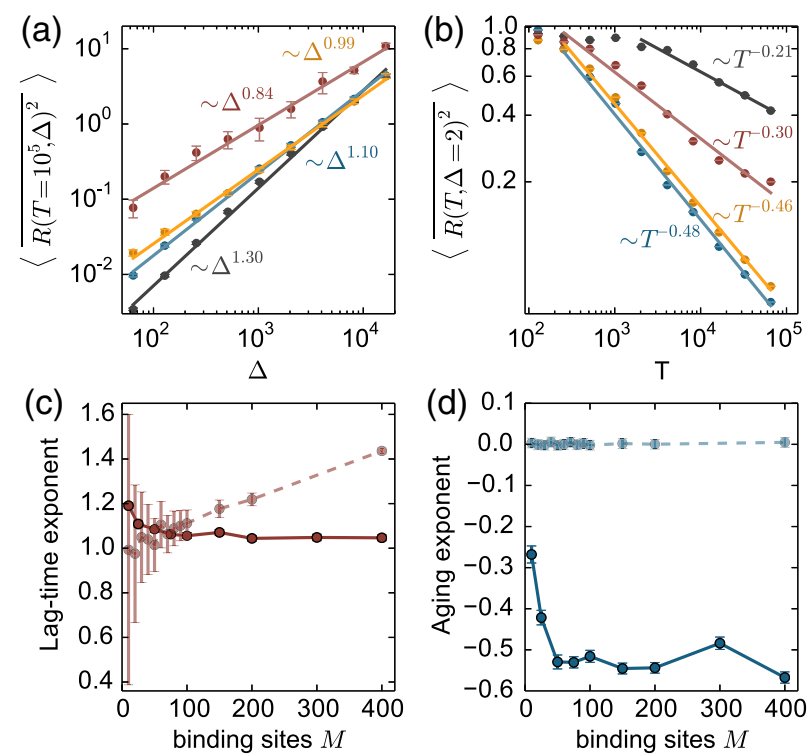

FIG. 3. Scaling in molecular simulations. (a) Time-averaged MSD as a function of lag time for binding radii $s=0.001$ (gray), 0.01 (blue), 0.1 (orange), and 1(red). (b) Mean-squared displacement as a function of measurement time for a range of binding radii [colors are the same as in (a)]. Each curve is rescaled to start at 1 for easier visualization. (c),(d) Dependence of the indicated exponents on the maximum number of possible attachments, $M$. The dashed lines show the outcome for a tug-of-war scenario, which can be obtained from our model by simulating the dynamics on two antiparallel filaments. The full lines show the exponents for the molecular simulations on a twodimensional random network. Lag-time exponents are calculated for $T=10^{5}$.

By varying the binding radius $s$ by factors of 10 from 0.001 to 1 , we can tune the transport from superdiffusive to subdiffusive. When the range of interaction is very small, the motor attaches only to the closest filament. As a result, the motion is superdiffusive but slow [note the intercept for the gray line in Fig. 3(a)] because the number of active attachments is low. For intermediate interaction ranges, the motor can simultaneously bind multiple filaments, and a tug-of-war-like mechanism gives rise to subdiffusive or diffusive motion.

We plot the MSD as a function of the measurement time $(T)$ in Fig. 3(b). For an ergodic system, the MSD should be constant as $T$ varies - the properties of the motion are the same independent of the length of recording. In contrast, a decrease in the MSD with $T$ suggests that there are longlived traps. The essential idea is that the traps increasingly dominate the statistics as more data are included in the averages. We observe a power-law decay (i.e., aging), consistent with prior analysis of myosin II on an in vitro actin network [10] and the motion of insulin granules in vivo $[9,10]$.

The observed aging exponent depends on the size of the binding radius $s$, but we obtain exponents comparable to 
experimental values as this parameter varies over 2 orders of magnitude. The molecular simulations of the model also predict more trapping for increased numbers of binding sites: both the lag-time and the aging exponents decrease with larger $M$ [Figs. 3(c) and 3(d)]. However, due to the nonergodic nature of this transport process, the lag-time exponent depends on the length of the recording $T$. We show the $T$ dependence of the exponents in Fig. S3 (see also Fig. S4) in Supplemental Material [30]. In contrast to the results that we obtain for motion on a random filament network, a simple tug-of-war scenario with a motor between two antiparallel filaments yields no glassy dynamics, independent of the number of binding sites. Also, the scaling of the MSD becomes increasingly superdiffusive with increased numbers of binding sites [Fig. 3(c)], and the diffusion constant increases (data not shown).

Having thus captured the statistics of experiments, we seek to use the model to elucidate the microscopic motions that underlie the statistics. In this regard, we notice that motors frequently exhibit vortexlike motions in which they steadily cycle from one filament to another at a junction [Fig. 4]. These motions persist for long times in comparison with the duration of the simulations. Cycling motions are of particular interest given that passive particles in a vortex flow in a fluid are known to exhibit power-law-distributed trapping times [41]. Analogous observations exist for trapped ions [42] and BoseEinstein condensates [43].

An approximate length scale for the vortices responsible for the glassy dynamics can be inferred from the crossover in the MSD curves. When the MSD as a function of lag time switches from superlinear to linear or sublinear scaling, $\Delta$ is sufficiently large for the MSD to include contributions from the vortices (Figs. S1 and S2 in Supplemental Material [30]). In other words, the MSD exponent decreases when the dynamics include bounded trajectories. In our conditions, the crossover occurs at $\Delta=100$, which corresponds to about $10 \mathrm{~s}$ based on the numbers for actin and myosin given above. In turn, for a

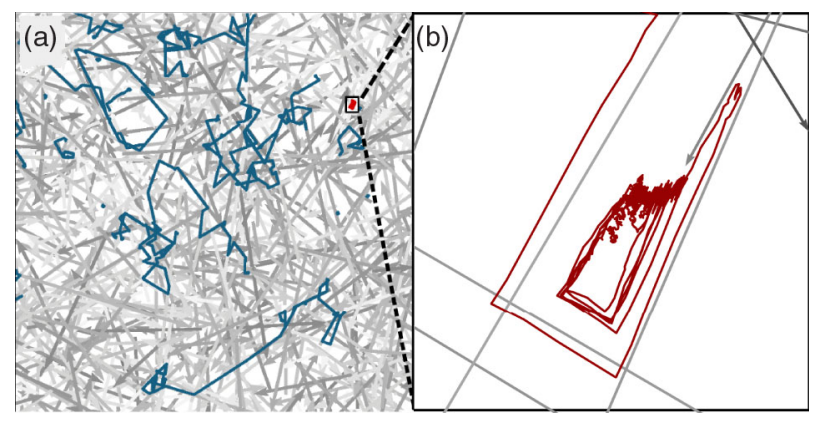

FIG. 4. Single-particle trajectories reveal a cycling state. (a) Representative simulation trajectories (blue and red) projected onto the filament network (gray). (b) A magnified view of a cycling trajectory (red). myosin II motor and the parameters in Table I, the length scale of vortices would be approximately $100 \mathrm{~nm}$.

\section{CYCLING STATE GIVES RISE TO POWER-LAW DWELL TIMES}

To investigate whether the observed vortexlike motion can account for the anomalous statistics, we now consider an idealized geometry. Specifically, we consider filaments that meet at right angles to form a square because it simplifies the mathematics (Fig. 5). However, we emphasize that the conclusions that we draw from this analysis are general: cycling can occur whenever the unit vectors of a group of crossing filaments sum to zero. In a square loop, the motor interacts with only one or two filaments at a time, and, due to the projection rule (Fig. 2), the motor always moves along the filament with the majority of sites bound. The average resulting motion can be described by
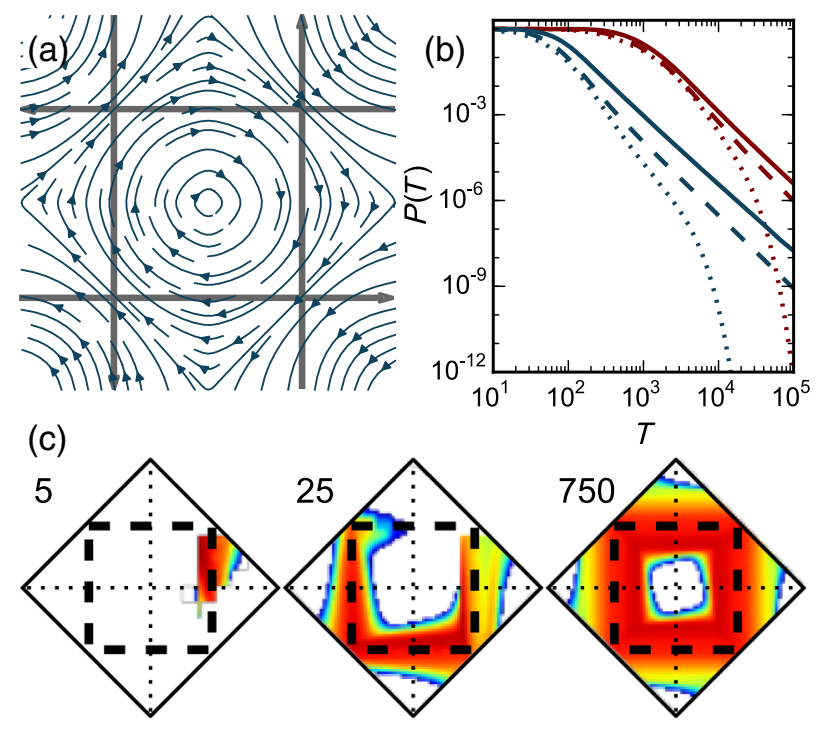

FIG. 5. Vortex model. (a) Streamlines (blue) for motion in the vicinity of an idealized square circuit of filaments (gray). (b) Survival probability in a vortex for $M=1$ (red) or $M=5$ (blue) in a vortex of size 40 . The probability is initialized to a nonzero value at $3 / 4$ of the diagonal and zero elsewhere. The binding radius is set to $1 / 5,1 / 7.5$, or $1 / 10$ of the vortex length (dotted, dashed, or full lines). The probability has three phases: constant, power law, and exponential. The length of the powerlaw decay decreases with increasing binding radius. (c) Numerical solution of the master equation in a square vortex. In the example shown, the initial condition is a localized probability at a single location similar to (b). Here, the vortex size is $40, M=5$, the binding radius is $1 / 10$ of the side length, and the motor step size is unit length. The colors correspond to the logarithm of the probability density of observing the motor at each location (red is highest) and is normalized in each subplot. The three panels show the master equation after 5, 25, and 750 iterations, respectively. The probability density spreads over time and the maximum rotates. The final image shows a pattern of localization that is stable as the integrated density continues to decrease. 
streamlines, which form a nested set of closed loops that do not cross [Fig. 5(a)]. If the dynamics were deterministic, the streamlines would describe the motion entirely, and the motor would stay in the vortex forever. However, due to the stochastic nature of binding and unbinding, individual trajectories deviate from the streamlines, and the motor eventually leaves the vortex.

To characterize this behavior quantitatively, we derive the master equation that governs this escape. For this purpose, we need to determine how the active binding sites distribute between the two accessible filaments via the two-step procedure described in Sec. II. The probability $P_{f}$ that $l_{1}$ out of $M$ possible attachment sites are assigned to filament 1 and that the remaining are assigned to filament 2 is binomial:

$$
P_{f}\left(l_{1}, l_{2}\right)=\left(\begin{array}{c}
M \\
l_{1}
\end{array}\right) \frac{b_{1}^{l_{1}} b_{2}^{l_{2}}}{\left(b_{1}+b_{2}\right)^{M}}, \quad \text { with } \quad l_{1}+l_{2}=M .
$$

The probability $P_{a}$ that $k_{1}$ out of $l_{1}$ possible attachments are active is also binomial:

$$
P_{a}\left(k_{1} \mid l_{1}\right)=\left(\begin{array}{l}
l_{1} \\
k_{1}
\end{array}\right) b_{1}^{k_{1}}\left(1-b_{1}\right)^{l_{1}-k_{1}}
$$

and similarly for $k_{2}$. Since filament assignment and selection of the active attachments are independent events, we can now write for the overall probability $P$ of having $k_{1}$ and $k_{2}$ active binding sites on filaments 1 and 2 :

$$
P\left(k_{1}, k_{2}\right)=\sum_{l_{1}=k_{1}}^{M-k_{2}} P_{f}\left(l_{1}, M-l_{1}\right) P_{a}\left(k_{1} \mid l_{1}\right) P_{a}\left(k_{2} \mid M-l_{1}\right) .
$$

The limits of the sum are set by $l_{1} \geq k_{1}, l_{2} \geq k_{2}$, and $l_{1}+l_{2}=M$.

Associating filament 1 with the $x$ direction and filament 2 with the $y$ direction, the resulting master equation for motion in a square vortex is

$$
\begin{aligned}
\frac{d P_{t}(x, y)}{d t}= & -\left(1-\sum_{k_{1}=k_{2}=0}^{M / 2} P\left(k_{1}, k_{2}\right)\right) P_{t-1}(x, y) \\
& +\sum_{k_{2}=0}^{M / 2-1} \sum_{k_{1}>k_{2}}^{M-k_{2}} P\left(k_{1}, k_{2}\right) P_{t-1}\left(x-k_{1}, y\right) \\
& +\sum_{k_{1}=0}^{M / 2-1} \sum_{k_{2}>k_{1}}^{M-k_{1}} P\left(k_{1}, k_{2}\right) P_{t-1}\left(x, y-k_{2}\right) .
\end{aligned}
$$

Here, $P_{t}(x, y)$ is the probability to be in a certain location $(x, y)$ in the vortex at time $t$, and we assume a unit time step. The first term accounts for motors that stay in place, while the second and third terms account for taking steps along filaments 1 and 2, respectively. Note that the polarity of the filaments is fixed, so that motion along each filament is unidirectional and no terms are needed to account for motion in the other direction. The vortex has absorbing boundaries where the velocity has a saddle point, forming a diamond-shaped region.

We solve this master equation numerically [Fig. 5(c)]. The survival probability in the vortex decays in three stages. At short times, the probability is close to unity, since the probability distribution needs a finite number of steps to reach the vortex boundaries. Then, we observe that the probability density also rotates within the vortex, similar to particles in the explicit simulations (see also Movie 1 in Supplemental Material [30]). During that time, the probability decays as a power law [see Fig. 5(b)]. The probability distribution in the vortex ultimately reaches a quasi-steady-state, when the shape of the distribution is no longer changing, and the survival probability decays as an exponential. The durations of these three stages are determined by the starting conditions and the relative size of the binding radius to the size of the vortex, as well as the number of binding sites. The power-law decay of the survival probability is longest for small binding radii (significantly smaller than the vortex) since the binding potential favors steps parallel to the filaments, and only allows very small steps orthogonal to the filaments. This relates to the results shown in Fig. 3(a) and 3(b), in that there exist a range of vortex sizes in our chosen network, which lead to characteristic trapping times. Binding radii much smaller or larger than these sizes will show less aging than midrange radii, due to the existing spatial scales in the random network. Relatedly, increasing the density of filaments in the network decreases the vortex size; this leaves the scaling with $\Delta$ unchanged while decreasing the extent of aging (Fig. S5 in Supplemental Material [30]).

The average motion in the vortex can be viewed as a combination of drift along the streamlines and diffusion orthogonal to the streamlines. From this perspective, the cycling motion is similar to that of a particle in a RayleighBénard convection cell [44]. However, the situation differs, in that the diffusion is position dependent [i.e., $P\left(k_{1}, k_{2}\right)$ varies in space through $b_{1}$ and $b_{2}$ ]. In other words, the master equation limits to a drift diffusion (Langevin) form with a multiplicative, rather than an additive, noise. This form reflects the fact that, in the motor model, the number of attachments is influenced by the position relative to the filaments defining the vortex boundary.

\section{EXPERIMENTAL DEMONSTRATION OF CYCLING-STATE CONTRIBUTIONS}

A key prediction of the cycling-state model is that the aging exponent decreases with the number of attachment sites on each motor [Fig. 3(d)]. We can test this idea without the confounding effects of cell signaling by studying mixtures of purified actin filaments and myosin motors. 
The specific system that we consider comprises actin filaments bundled by the passive cross-linker fimbrin. The motors are minifilaments of skeletal muscle myosin II, which polymerizes into large assemblies with on the order of 100 motor proteins [45]. The actin and myosin molecules are visualized through fluorescence microscopy, as detailed in the Supplemental Material [30]. Singleparticle trajectories are obtained by tracking, and trajectories with fewer than 30 time points are discarded. There are 246 resulting trajectories with a mean length of $166 \mathrm{~s}$, with a standard deviation of $120 \mathrm{~s}$. This length is long compared with typical in vitro particle-tracking studies of isolated motors on single filaments. Both the large number of heads and the density of binding sites in the filament network make it unlikely for motors to detach, which favors processivity. The MSD as a function of measurement time $T$ shows aging, with an exponent comparable to previously published data (compare Ref. [10] with Figs. S6A and S6B in Supplemental Material [30]).

To test the prediction in Fig. 3(d), we exploit the fact that the number of myosin molecules in each minifilament varies naturally and use fluorescence intensity as a proxy for the number of motor heads. We divide the trajectories into two groups according to the median fluorescence of all trajectories. There are 123 single-particle trajectories for motors with relatively high intensity and an equal number of trajectories for motors with relatively low intensity. We expect motors with higher intensity to have more heads and thus exhibit stronger aging. We observe that this is the case [Fig. 6(a)]. Specifically, we use case resampling (a form of bootstrapping) to get a distribution of exponents from each fluorescence group. The means of these distributions are $-0.6125 \pm 0.0035$ and $-0.5112 \pm 0.0086$ (mean \pm SEM high-fluorescence and low-fluorescence groups, respectively). We test whether the means are significantly different using the unequal variances $t$-test. The two-tailed $p$ value is less than $10^{-4}$, indicating that the difference is significant.

Additionally, we manually select trajectories that visibly cycle (loop over the same multipixel region more than once). Twenty-nine trajectories are found to cycle, and a significant portion of these are also classified as high fluorescence $[N=22, p$ value $=0.00185$, Fisher's exact test for cycling (noncycling) versus high (low) fluorescence]. Because the actin filaments do not move significantly (Fig. S7 in Supplemental Material [30]), we can project trajectories onto the network, and we see that the trajectories that result in the most pronounced decay have visible cycling that coincides with filament junctions [arrows in Figs. 6(b)-6(d)].

While the observations above demonstrate the existence of cycling, we seek to exclude alternative mechanisms for the statistical differences in exponents in Fig. 6(a). Reasonable candidates are intrinsic differences in motor speeds and detachment. With regard to the former, we note

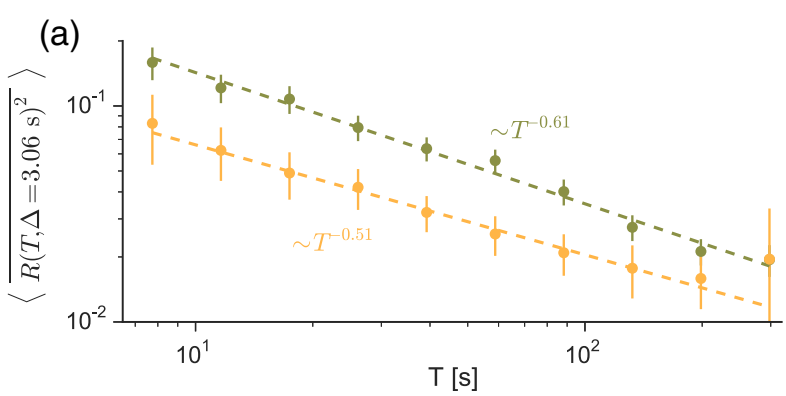

(b)

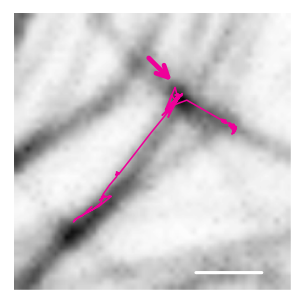

(c)

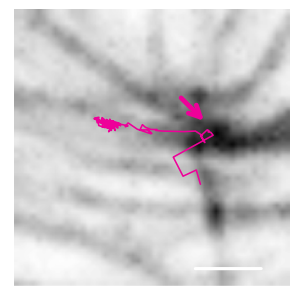

(d)

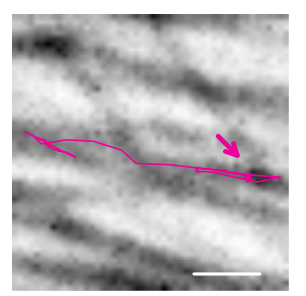

(e)

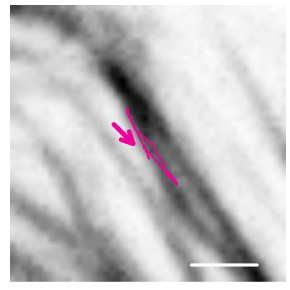

FIG. 6. Experimental trajectories show cycling. (a) Trajectories are divided into two groups according to the total fluorescence intensity of the motor. The group with higher fluorescence intensity (green) shows a larger aging exponent than the lowfluorescence group (yellow). (b)-(e) Representative trajectories of individual myosin II minifilaments overlayed onto the actin network. Arrows denote cycling events. Trajectories are obtained from myosin II minifilaments on an actin network bundled with fimbrin. Scale bar is $1.1 \mu \mathrm{m}$, trajectories shown are imaged at 1.5 s intervals; see Supplemental Material for details [30]. Singleparticle trajectories are obtained using the Python-based implementation of the Crocker-Grier algorithm TRACKPY [46].

that the average motor step size from frame to frame (i.e., the net speed for movement over the filament network) does not differ for the high- and low-intensity groups: the mean $\pm \mathrm{SEM}$ are $44.1 \pm 2.6$ and $45.0 \pm 2.5 \mathrm{~nm} / \mathrm{s}$, respectively. If detachment were instead responsible for the anomalous dynamics, the particles should undergo simple diffusion when apparently trapped. To test for this possibility, we divide the trajectories between trapped and nontrapped periods and then analyze their dynamics using a published measure for detecting complex dynamics at resolutions of only a few frames [10]. We find that the motion is not simple diffusion, as detailed in the Supplemental Material (see also Fig. S9) [30].

Together, these results strongly support the idea that the cycling state exists for motors with multiple attachments and gives rise to power-law decay in the MSD as a function of measurement time. 


\section{DISCUSSION}

Motor-driven processes in cells often exhibit anomalous statistics [12,47-49], and these statistics can have important functional consequences [9]. While existing random-walk models of aging typically start by assuming trapped states with power-law dwell times, we introduce a microscopic mechanism for how simple biologically consistent interactions can give rise to such long-lived traps. The glassy dynamics result from a vortexlike state that emerges in multiple dimensions-motors that can attach to multiple filaments simultaneously cycle unproductively at filament junctions, and the escape times from these flows are powerlaw distributed over a time range set by the motor step size and the filament spacing. We demonstrate that such cycling events occur frequently in the motion of skeletal myosin II assemblies on a dense, random network of bundles of actin filaments in vitro, and we use this system to validate the predictions of the model. To the best of our knowledge, these cycling dynamics have not been appreciated previously, and we expect that their topological features will lead to rich physics beyond idealized trap models.

Microtubule structures with many intersections are observed above the basal membrane of epithelial cells, where they function in endocytic vesicle transport [50]. A study that reconstructed these epithelial microtubule networks in vitro observed a kinesin-coated bead cycling through a vortex structure [51]. The same study (and others [52]) also saw a slowdown or pausing states at intersections, which is also present in our model due to the motors interacting with both filaments at the same time. While these structures are macroscopic compared to the vortices responsible for the observed glassy behavior, they show that motor-associated cargo or multibinding complexes can navigate intersections and cycles for certain geometries.

Tug-of-war models [24] contain similar molecular elements and exhibit pauses when motors are stalling or opposing forces match exactly. However, such models are essentially one dimensional and do not give rise to powerlaw-distributed dwell times. Based on Figs. 3(c) and 3(d), we expect the cycling state to be prevalent only when motor assemblies comprise many protein motors and directed motion dominates over thermal processes. Indeed, measured exponents for the MSD could be used to infer the size of such assemblies. However, care is needed because different filament structures favor different amounts of directed, tug-of-war, and vortexlike motions. Random networks in three dimensions have few circuits that lead to cycling, while cytoskeletal networks that are organized by specific filament binding proteins (e.g., Arp2/3), as well as quasi-twodimensional networks that arise in cell cortices, have larger numbers of suitable junctions. Thus, accurate estimates of the numbers of active motor heads requires constructing a calibration curve for the exponent for each network structure.

Understanding how motor assemblies behave on complex filament networks in cells is an outstanding challenge
[53]. The degree to which the cycling state contributes to dynamics in different contexts in vivo is an open question deserving further study. Cells could potentially spatiotemporally control intracellular transport by rearranging their cytoskeletal networks to favor or disfavor cycling. Understanding how this control mechanism manifests in different types of cells and tissue environments, as well as its interplay with other regulatory processes and trapping mechanisms [25], is a useful direction for future research. It will also be interesting to understand the interplay of motor transport, force transmission, and network rearrangement.

\section{ACKNOWLEDGMENTS}

We thank Samantha Stam and David Kovar's laboratory for protein purification; Michael Murrell, Jennifer Ross, Toan Huynh, and Norbert Scherer for helpful conversations; and Glen Hocky and Shiladitya Banerjee for critical readings of the manuscript. Support was provided by the University of Chicago Materials Research Science and Engineering Center (NSF DMR-1420709) and the W. M. Keck Foundation.

[1] I. Y. Wong, M. L. Gardel, D. R. Reichman, E. R. Weeks, M. T. Valentine, A. R. Bausch, and D. A. Weitz, Anomalous Diffusion Probes Microstructure Dynamics of Entangled F-Actin Networks, Phys. Rev. Lett. 92, 178101 (2004).

[2] B. Wang, S. M. Anthony, S. C. Bae, and S. Granick, Anomalous yet Brownian, Proc. Natl. Acad. Sci. U.S.A. 106, 15160 (2009).

[3] B. R. Parry, I. V. Surovtsev, M. T. Cabeen, C. S. O’Hern, E. R. Dufresne, and C. Jacobs-Wagner, The Bacterial Cytoplasm Has Glass-like Properties and Is Fluidized by Metabolic Activity, Cell 156, 183 (2014).

[4] Y. Sako, S. Minoghchi, and T. Yanagida, Single-Molecule Imaging of EGFR Signalling on the Surface of Living Cells, Nat. Cell Biol. 2, 168 (2000).

[5] D. Cai, D. P. McEwen, J. R. Martens, E. Meyhofer, and K. J. Verhey, Single Molecule Imaging Reveals Differences in Microtubule Track Selection between Kinesin Motors, PLoS Biol. 7, e1000216 (2009).

[6] S. B. Zimmerman and S. O. Trach, Estimation of Macromolecule Concentrations and Excluded Volume Effects for the Cytoplasm of Escherichia coli, J. Mol. Biol. 222, 599 (1991).

[7] M. C. Konopka, I. A. Shkel, S. Cayley, M. T. Record, and J. C. Weisshaar, Crowding and Confinement Effects on Protein Diffusion In Vivo, J. Bacteriol. 188, 6115 (2006).

[8] F. Höfling and T. Franosch, Anomalous Transport in the Crowded World of Biological Cells, Rep. Prog. Phys. 76, 046602 (2013).

[9] S. M. A. Tabei, S. Burov, H. Y. Kim, A. Kuznetsov, T. Huynh, J. Jureller, L. H. Philipson, A. R. Dinner, and N. F. Scherer, Intracellular Transport of Insulin Granules Is a Subordinated Random Walk, Proc. Natl. Acad. Sci. U.S.A. 110, 4911 (2013). 
[10] S. Burov, S. M. A. Tabei, T. Huynh, M. P. Murrell, L. H. Philipson, S. A. Rice, M. L. Gardel, N. F. Scherer, and A. R. Dinner, Distribution of Directional Change as a Signature of Complex Dynamics, Proc. Natl. Acad. Sci. U.S.A. 110, 19689 (2013).

[11] I. Golding and E. C. Cox, Physical Nature of Bacterial Cytoplasm, Phys. Rev. Lett. 96, 098102 (2006).

[12] I. M. Tolić-Nørrelykke, E.-L. Munteanu, G. Thon, L. Oddershede, and K. Berg-Sørensen, Anomalous Diffusion in Living Yeast Cells, Phys. Rev. Lett. 93, 078102 (2004).

[13] S. C. Weber, A. J. Spakowitz, and J. A. Theriot, Bacterial Chromosomal Loci Move Subdiffusively through a Viscoelastic Cytoplasm, Phys. Rev. Lett. 104, 238102 (2010).

[14] A. V. Weigel, B. Simon, M. M. Tamkun, and D. Krapf, Ergodic and Nonergodic Processes Coexist in the Plasma Membrane as Observed by Single-Molecule Tracking, Proc. Natl. Acad. Sci. U.S.A. 108, 6438 (2011).

[15] P. C. Bressloff and J. M. Newby, Stochastic Models of Intracellular Transport, Rev. Mod. Phys. 85, 135 (2013).

[16] L. F. Cugliandolo, J. Kurchan, and F. Ritort, Evidence of Aging in Spin-Glass Mean-Field Models, Phys. Rev. B 49, 6331 (1994).

[17] C. Manzo, J. A. Torreno-Pina, P. Massignan, G. J. Lapeyre, Jr., M. Lewenstein, and M. F. Garcia Parajo, Weak Ergodicity Breaking of Receptor Motion in Living Cells Stemming from Random Diffusivity, Phys. Rev. X 5, 011021 (2015).

[18] S. Seino, T. Shibasaki, and K. Minami, Dynamics of Insulin Secretion and the Clinical Implications for Obesity and Diabetes, J. Clin. Invest. 121, 2118 (2011).

[19] J.-P. Bouchaud and D. S. Dean, Aging on Parisi's Tree, J. Phys. I (France) 5, 265 (1995).

[20] M. V. Feigel'man and V. M. Vinokur, On the Stochastic Transport in Disordered Systems, J. Phys. (Paris) 49, 1731 (1988).

[21] J. A. Dix and A. S. Verkman, Crowding Effects on Diffusion in Solutions and Cells, Annu. Rev. Biophys. 37, 247 (2008).

[22] A. Varadi, T. Tsuboi, L. I. Johnson-Cadwell, V. J. Allan, and G. A. Rutter, Kinesin I and Cytoplasmic Dynein Orchestrate Glucose-Stimulated Insulin-Containing Vesicle Movements in Clonal MIN6 $\beta$-Cells, Biochem. Biophys. Res. Commun. 311, 272 (2003).

[23] R. D. Vale, F. Malik, and D. Brown, Directional Instability of Microtubule Transport in the Presence of Kinesin and Dynein, Two Opposite Polarity Motor Proteins., J. Cell Biol. 119, 1589 (1992).

[24] M. J. I. Müller, S. Klumpp, and R. Lipowsky, Tug-of-War as a Cooperative Mechanism for Bidirectional Cargo Transport by Molecular Motors, Proc. Natl. Acad. Sci. U.S.A. 105, 4609 (2008).

[25] J. Newby and P.C. Bressloff, Local Synaptic Signaling Enhances the Stochastic Transport of Motor-Driven Cargo in Neurons, Phys. Biol. 7, 036004 (2010).

[26] A. G. Hendricks, E. Perlson, J. L. Ross, H. W. Schroeder III, M. Tokito, and E. L. F. Holzbaur, Motor Coordination via a Tug-of-War Mechanism Drives Bidirectional Vesicle Transport, Curr. Biol. 20, 697 (2010).

[27] V. Soppina, A. K. Rai, A. J. Ramaiya, P. Barak, and R. Mallik, Tug-of-War between Dissimilar Teams of Microtubule Motors Regulates Transport and Fission of
Endosomes, Proc. Natl. Acad. Sci. U.S.A. 106, 19381 (2009).

[28] M. Y. Ali, G. G. Kennedy, D. Safer, K. M. Trybus, H. L. Sweeney, and D. M. Warshaw, Myosin Va and Myosin VI Coordinate Their Steps while Engaged in an In Vitro Tug of War during Cargo Transport, Proc. Natl. Acad. Sci. U.S.A. 108, E535 (2011).

[29] W. O. Hancock, Bidirectional Cargo Transport: Moving beyond Tug of War, Nat. Rev. Mol. Cell Biol. 15, 615 (2014).

[30] See Supplemental Material at http://link.aps.org/ supplemental/10.1103/PhysRevX.6.011037 for alternative models, experimental details, and further analysis of trapped states.

[31] R. H. Lee and C. S. Mitchell, Axonal Transport Cargo Motor Count versus Average Transport Velocity: Is Fast versus Slow Transport Really Single versus Multiple Motor Transport?, J. Theor. Biol. 370, 39 (2015).

[32] J. T. Finer, R. M. Simmons, and J.A. Spudich, Single Myosin Molecule Mechanics: Piconewton Forces and Nanometre Steps, Nature (London) 368, 113 (1994).

[33] J. Howard, Molecular Motors: Structural Adaptations to Cellular Functions, Nature (London) 389, 561 (1997).

[34] P. Pierobon, S. Achouri, S. Courty, A. R. Dunn, J. A. Spudich, M. Dahan, and G. Cappello, Velocity, Processivity, and Individual Steps of Single Myosin V Molecules in Live Cells, Biophys. J. 96, 4268 (2009).

[35] M. J. Saxton and K. Jacobson, Single-Particle Tracking: Applications to Membrane Dynamics, Annu. Rev. Biophys. Biomol. Struct. 26, 373 (1997).

[36] J.-P. Bouchaud, Weak Ergodicity Breaking and Aging in Disordered Systems, J. Phys. I (France) 2, 1705 (1992).

[37] A. Lubelski, I. M. Sokolov, and J. Klafter, Nonergodicity Mimics Inhomogeneity in Single Particle Tracking, Phys. Rev. Lett. 100, 250602 (2008).

[38] Y. He, S. Burov, R. Metzler, and E. Barkai, Random TimeScale Invariant Diffusion and Transport Coefficients, Phys. Rev. Lett. 101, 058101 (2008).

[39] I. M. Sokolov, Viewpoint: Statistics and the Single Molecule, Physics 1, 8 (2008).

[40] J. H. P. Schulz, E. Barkai, and R. Metzler, Aging Effects and Population Splitting in Single-Particle Trajectory Averages, Phys. Rev. Lett. 110, 020602 (2013).

[41] T. H. Solomon, E. R. Weeks, and H. L. Swinney, Observation of Anomalous Diffusion and Lévy Flights in a TwoDimensional Rotating Flow, Phys. Rev. Lett. 71, 3975 (1993).

[42] R. G. DeVoe, Power-Law Distributions for a Trapped Ion Interacting with a Classical Buffer Gas, Phys. Rev. Lett. 102, 063001 (2009).

[43] E. Lundh and P. Ao, Hydrodynamic Approach to Vortex Lifetimes in Trapped Bose Condensates, Phys. Rev. A 61, 063612 (2000).

[44] B. I. Shraiman, Diffusive Transport in a Rayleigh-Bénard Convection Cell, Phys. Rev. A 36, 261 (1987).

[45] T. Thoresen, M. Lenz, and M. L. Gardel, Thick Filament Length and Isoform Composition Determine Self-Organized Contractile Units in Actomyosin Bundles, Biophys. J. 104, 655 (2013).

[46] D. B. Allan, T. A. Caswell, and N. C. Keim, TRACKPY v0.2, (2014). 
[47] M. Weiss, M. Elsner, F. Kartberg, and T. Nilsson, Anomalous Subdiffusion Is a Measure for Cytoplasmic Crowding in Living Cells, Biophys. J. 87, 3518 (2004).

[48] F. Amblard, A. C. Maggs, B. Yurke, A. N. Pargellis, and S. Leibler, Subdiffusion and Anomalous Local Viscoelasticity in Actin Networks, Phys. Rev. Lett. 77, 4470 (1996).

[49] J.-H. Jeon, V. Tejedor, S. Burov, E. Barkai, C. SelhuberUnkel, K. Berg-Sørensen, L. Oddershede, and R. Metzler, In Vivo Anomalous Diffusion and Weak Ergodicity Breaking of Lipid Granules, Phys. Rev. Lett. 106, 048103 (2011).

[50] A. Reilein, S. Yamada, and W. J. Nelson, SelfOrganization of an Acentrosomal Microtubule Network at the Basal Cortex of Polarized Epithelial Cells, J. Cell Biol. 171, 845 (2005).

[51] J. L. Ross, H. Shuman, E. L.F. Holzbaur, and Y.E. Goldman, Kinesin and Dynein-Dynactin at Intersecting Microtubules: Motor Density Affects Dynein Function, Biophys. J. 94, 3115 (2008).

[52] O. Osunbayo, J. Butterfield, J. Bergman, L. Mershon, V. Rodionov, and M. Vershinin, Cargo Transport at Microtubule Crossings: Evidence for Prolonged Tug-of-War between Kinesin Motors, Biophys. J. 108, 1480 (2015).

[53] M. W. Elting and J. A. Spudich, Future Challenges in Single-Molecule Fluorescence and Laser Trap Approaches to Studies of Molecular Motors, Dev. Cell 23, 1084 (2012). 